\title{
APRENDIZAJE POR PROYECTOS
}

\author{
Paola Vanessa Ubillus Salas
}

\begin{abstract}
RESUMEN
El presente artículo tiene por finalidad mostrar la relevancia de la enseñanza por proyectos para materializar los objetivos de las diversas propuestas pedagógicas, ya que las exigencias del mundo actual requieren desarrollar habilidades de investigación para poder indagar, organizar y condensar mucha información que finalmente sea relevante 0 significativa para el estudiante y lo pueda aplicar en la solución de un aspecto en la realidad. Esta oportunidad nos la dan los proyectos de aprendizaje y para ello los docentes debemos estar no sólo metodológicamente preparados sino conocer como funcionan las sinergias en el aula de clases.
\end{abstract}

\section{PALABRAS CLAVE}

Proyectos, habilidades, Investigación, metodología, realidad educativa.

\section{ABSTRACT}

The present article aims to show the importance of teaching by projects to materialize the objectives of the several pedagogical proposals, since the demands of today's world require developing research skills to be able to investigate, organize and summarize a lot of information that is finally relevant or significant for the student and can apply it in the solution of an aspect in reality. This opportunity gives us the learning projects and for them, teachers must be not only methodologically prepared but also know how synergies work in the classroom.

\section{KEYWORDS}

Projects, skills, research, methodology, educational reality.
S e dice que una de las formas de felicidad es trabajar en aquello que nos despierta pasión, por lo que con el tiempo, muchos docentes que entramos a un aula con la finalidad de despertar esa pasión en nuestros alumnos por aquel tema o área del conocimiento que le llevamos, nos encontramos que la clase mejor preparada no causa el efecto que esperábamos y los estudiantes no se identifican con lo que les compartimos, y solo con el tiempo nos damos cuenta que el camino no es esa mirada vertical de creer que somos muy expertos en ese tema, sino que el éxito de los procesos educativos está en ser expertos en estrategias que evidencien que lo que mi alumno está aprendiendo conmigo es significativo para él, y ser significativo es que se conecta con su realidad, se conecta con lo que necesita para solucionar problemas en su día a día, no busco que se vuelva un experto en la materia pero sí que esas capacidades que mi materia moviliza en él le sirva para la vida, para el trabajo, para relacionarse mejor con su entorno y, sobre todo, que actitudinalmente llegue a ser una persona equilibrada y valiosa para su grupo de referencia y para su sociedad.

La respuesta a todos nuestros esfuerzos por hacer del colegio un lugar motivante, expectante para nuestros alumnos, está en conectarnos a su natural impulso indagador, el cual es innato, todos exploramos desde que llegamos al mundo, nuestro día a día es explorar el entorno, y aunque muchas teorías han intentado explicar cómo nos adueñamos del mundo, lo concreto es que el instinto de supervivencia descansa sobre la adaptación en su sentido más básico. Si Piaget nos dice que vamos armando estructuras de aprendizaje entre lo que tenemos y lo nuevo, lo ideal sería poder 
guiar esa construcción, pero de tal forma que nuestro estudiante sea consciente de este proceso, reconozca cuáles son sus caminos para aprender y sobre todo nunca quiera dejar de hacerlo porque lo motiva saber más acerca de algo. Aquí es donde entramos al campo de la motivación, sin ella, ese componente actitudinal que consolida lo lógico racional, el aprendizaje, se volvería vano y efímero. Los docentes de las especialidades más abstractas como las ciencias y las matemáticas ya están volteando la mirada a ese factor emocional que hará que su estudiante no sufra con las ciencias y los números el resto de su vida, o por el contario, los sienta presenta de manera explícita en su día a día y sienta la importante que es indagar al respecto sin sufrimiento, sólo por el placer de solucionar su cotidianeidad desde los conocimientos que se le imparte.

Un individuo transcurre en promedio veinte años de su vida en las aulas y con el objetivo de ser exitoso y ser competente en aquello que nos hemos propuesto, y ser competentes es saber actuar de la forma como el entorno y la circunstancia nos exige y para lograrlo es necesario que todos nuestros conocimientos interactúen. Una sola materia, un solo punto de vista, una sola forma de ver las cosas de manera aislada no nos dará el camino; pero, si por el contrario, los docentes estimulamos en nuestros estudiantes la integración de saberes, conocimientos, experiencias, capacidades, habilidades y destrezas en torno a algo, que los motiva como individuos, como equipo, y hace que todo esos conocimientos se integren, se movilicen y eso acompañado de la idoneidad en las relaciones interpersonales, promoviendo el trabajo en equipo, y siempre con una mirada sobre cuestiones éticas, entonces tendremos esa forma de enseñar que no es sólo "van a aprender conmigo" sino es "vamos a aprender juntos".

Y así es como los proyectos de aprendizaje adquieren total importancia, vuelve a la pedagogía ya no una fuente de conocimientos sino una fuente de capacidades para aprender en el sentido más funcional y emotivo posible.

El objetivo principal de las propuestas pedagógicas es el desarrollo de competencias "Un saber actuar en un contexto particular, donde el estudiante selecciona y moviliza de manera pertinente e integrada una diversidad de saberes, conocimientos y habilidades propios y recursos externos, para resolver una situación problemática o lograr un propósito determinado, satisfaciendo ciertos criterios de acción considerados esenciales" (MINEDU 2013), y si analizamos la nueva propuesta del Currículo Nacional 2016, encontramos la siguiente definición: "La competencia se define como la facultad que tiene una persona de combinar un conjunto de capacidades a fin de lograr un propósito específico en una situación determinada, actuando de manera pertinente y con sentido ético. Ser competente supone comprender la situación que se debe afrontar y evaluar las posibilidades que se tiene para resolverla. Esto significa identificar los conocimientos y habilidades que uno posee o que están disponibles en el entorno, analizar las combinaciones más pertinentes a la situación y al propósito, para luego tomar decisiones; y ejecutar o poner en acción la combinación seleccionada" (MINEDU 2016).

Por lo que el aprendizaje por proyectos supone generar en nuestros alumnos las capacidades necesarias para abordar esta forma de enseñanza que ha cobrado importancia cuando hablamos de "innovación educativa", ya que le da un papel mucho más activo al alumno y nos supone una reorganización no sólo del aula como espacio de aprendizaje sino de nuestros propios conceptos, estrategias y enfoques educativos.

Los proyectos activan la motivación de nuestros alumnos ya que integran varios conocimientos y los organizan de forma secuencial, de tal manera que la reflexión está presente en cada momento y les otorga autonomía en su proceso de aprendizaje. Y para que todo esto se consolide, es imprescindible una PLANIFICACIÓN por parte del docente donde los objetivos y metas a lograr estén sumamente claros y pueda guiar a su alumno para poder llegar a ellos, y canalizar e incorporar las preguntas, inquietudes y nuevos conocimientos. Esta planificación supone una secuencia definida desde lo que necesitamos previamente para afrontar esta construcción tanto a nivel de capacidades cognitivas, 
conocimientos previstos, hasta recursos materiales que permitan materializar lo planeado.

Se define un proyecto de aprendizaje como: "Una forma de planificación integradora que permite desarrollar competencias en los estudiantes, con sentido holístico e intercultural, promoviendo su participación en todo el desarrollo del proyecto. Comprende además procesos de planificación, implementación, comunicación y evaluación de un conjunto de actividades articuladas, de carácter vivencial o experiencial, durante un periodo de tiempo determinado, según su propósito, en el marco de una situación de interés de los estudiantes o problema del contexto" (MINEDU 2013).

La actitud del docente al llevar adelante proyectos de aprendizaje, es una actitud de apertura, ya que los estudiantes son quienes manifiestan sus intereses y necesidades e incluso señalan algún aspecto de la realidad que les resulta problemático y que buscan resolver, y este empoderamiento del estudiante dentro de su propio proceso de aprendizaje, promueve además el trabajo colaborativo, la perseverancia y la comunicación asertiva.

Tener una actitud de apertura en el docente, implica además una mirada vigilante para ir incorporando determinadas actividades que nos pueda evidenciar el avance de los proyectos; y que todo lo desarrollado sea coherente a la meta planteada. La investigación cobra protagonismo durante todo el desarrollo de un proyecto de aprendizaje, la gestión de la información que resulte significativa para nuestro proyecto, el manejo de las fuentes y los procesos cognitivos que genera, en torno al tratamiento de dicha información, promueve en nuestros alumnos una serie de capacidades que finalmente no resultan aisladas solo a ese proyecto que se desarrolla, sino que se extrapola a otros campos y espacios de aprendizaje, volviendo a las asignaturas ya no aisladas sino vinculadas e interconectadas. Si se traslada esas situaciones en aula a la vida cotidiana, veremos que, resolver problemas no se logra aislando conocimientos sino integrando una serie de saberes para tomar decisiones, desarrollar habilidades, y finalmente llegar a la mejor opción posible.
No se puede dejar de relacionar la enseñanza por proyectos a la estimulación de la inteligencia emocional; la misma interacción del grupo, sustentación de puntos de vista, genera un espacio democrático donde los estudiantes pueden ver la utilidad pragmática de cada uno de los conocimientos que la escuela nos brinda, ello sin contar que ver nuestro entorno como fuente de conocimientos que retornarán en forma de soluciones, propuestas, estrategias etc. Y si hablamos a nivel actitudinal es innegable el proceso de sensibilización que se da en nuestros estudiantes, se vuelven activos, se involucran en la problemática de su comunidad más cercana y hace que tomen interés sobre cuestiones de importancia global, que si alguna vez las sintieron lejanas, por medio de los proyectos se llega a ver el impacto que ellas tienen en nuestra cotidianidad.

Y para tener un norte claro es necesario hacernos preguntas claves para que nuestras propuestas de proyectos de aprendizaje sean reales, posibles en el tiempo y sobretodo valiosas para nuestros alumnos dentro de sus procesos de aprendizaje. ¿Qué quiero que aprendan mis estudiantes? ¿Cómo se relaciona con el perfil, el currículo etc.? ¿Las actividades propuestas serán pertinentes, lograrán el efecto deseado? ¿Movilizarán cognitiva y emocionalmente a mis estudiantes? ¿Qué dificultades se podrían presentar en el camino y cómo los superaremos como equipo?

Un espacio de aprendizaje por proyectos posee una palabra clave presente en la mente de un docente: FLEXIBILIDAD, la secuencia de acciones y actividades en un proyecto de esta naturaleza está sujeto a una serie de variables que puede modificar el rumbo, el sentido y las motivaciones de nuestros estudiantes, pero a partir de las estrategias del docente, el escenario más oscuro dentro del desarrollo de un proyecto se puede volver una oportunidad de trabajar más capacidades, crear nuevas habilidades y sobretodo enrumbar hacia la meta a nuestros alumnos al reorientar el proceso.

Las VENTAJAS del aprendizaje por proyectos se evidencian en lo cotidiano, pero además sus ventajas a largo plazo se notan cuando se aplican las competencias en otros espacios que van más allá de lo académico. Estas ventajas principian desde la superación de esquemas educativos tradicionales, que son superados por pautas que hacen del 
aprendizaje una experiencia motivadora y desafiante para el estudiante, lo acerca afectivamente al aula como un espacio creativo, productivo y sobre todo cercano a lo que considera tangible, conocido en su entorno. Otra ventaja significativa es lo referente al trabajo en equipo; la interacción entre los estudiantes se basa en relaciones de respeto, equidad y sobre todo de apertura hacia las opiniones y aportes de los demás y al instaurarse como una pauta de interrelación, con el tiempo y ya en el plano profesional podrá afianzarse como un profesional proactivo y un elemento que sumará a sus equipos de trabajo donde le toque desenvolverse. En una sociedad globalizada, interconectada, la habilidad de coordinar con equipos, aportar, ya es un valor agregado, y si a ello le sumamos poder integrar información en un ejercicio deductivo que genere nuevo conocimiento, es un aporte que los proyectos de aprendizaje pueden afianzar en los perfiles educativos desde varios enfoques. No se trata solo de una mirada ecléctica sino de integrar con coherencia mucha información que vuelva el acto de aprender en algo cotidiano y natural y no solo reservado para ambientes académicos.

El estudiante llega a identificar su ritmo y estrategias para aprender y lograr tener una visión clara de su propio valor como agente productivo, refuerza su autoestima y sabe cómo aportar desde el rol o función que le ha tocado desarrollar dentro de un equipo. Y el establecer estas sinergias lo conlleva a mejorar su productividad y la de equipo.

En los proyectos de aprendizaje, además de afianzar conocimiento, profundizar en la observación del entorno genera una sensibilidad que pasa primordialmente por una pauta ética, no es el conocimiento sin la mirada del otro para el otro, los equipos se manejan entorno al respeto y escucha activa dentro de ellos y con mayor razón fuera de ellos. Los proyectos promueven destrezas, habilidades y conocimientos, pero a la vez la consolidación de valores que surgen en el mismo grupo y se proyectan a manejar estos códigos en todos los espacios donde le toque desarrollarse a nuestro estudiante.

Los proyectos de aprendizaje por su carácter FLEXIBLE requieren de la participación de capacidades y habilidades que los estudiantes adquieren en otras asignaturas, y se transversaliza de manera consiente, ya que cada actividad del proyecto requiere que se focalice en un aspecto de la realidad y para ello no se puede pretender que una sola asignatura explique fenómenos tan complejos como los que se dan en nuestro contexto. Esta integración de conocimientos responde a su vez del resultado de varias "formas de aprender", algo así como lo que Gardner define en su teoría de "Inteligencias Múltiples", las cuales no se constituyen de manera unilateral sino que establecen relaciones afines para afrontar determinadas situaciones. Gardner señalaba que la Inteligencia al ser un proceso dinámico, establece sinergias en torno a la obtención y procesamiento de la información y también se ven evidenciadas a nivel del equipo del proyecto. Cada integrante aporta desde su tipo de inteligencia predominante y es así como de diversos estilos marcar la tendencia de investigación que posee cada proyecto. La confluencia de estos puntos de vista influye desde la idea general hasta la esencia del objetivo o meta a lograr.

Finalmente, si un estudiante desarrolla habilidades investigativas y además integra todo ese conocimiento en estructuras de información organizadas de tal manera que sea funcional y aplicable a su realidad, los proyectos de aprendizaje habrán cumplido su objetivo, ya que la globalización ha puesto a nuestro alcance mucha información por lo que los objetivos primordiales de la educación ya no serán impartir conocimientos sino gestionarlos.

\section{REFERENCIAS}

Batista J., Enrique E. (2007). Lineamientos pedagógicos para la enseñanza y el aprendizaje. Colombia: Ed. Universidad Cooperativa de Colombia.

MINEDU (2013). Rutas de Aprendizaje - Los proyectos de Aprendizaje para el Logro de Competencias. Lima: Cimagraf.

Suazo Díaz, Sonia (2006). Inteligencia Múltiple: Manual Práctico para nivel Elemental. Ed. Universidad de Puerto Rico. 